\title{
Impact of a preoperative pharmaceutical consultation in scheduled orthopedic surgery on admission: a prospective observational study
}

Amélie Renaudin ${ }^{*}$, Géraldine Leguelinel-Blache ${ }^{1,2,3}$, Chloé Choukroun ${ }^{1}$, Audrey Lefauconnier ${ }^{4}$, Christophe Boisson ${ }^{4}$, Jean-Marie Kinowski ${ }^{1,2}$, Philippe Cuvillon ${ }^{4,5}$ and Hélène Richard ${ }^{1}$

\begin{abstract}
Background: Medication errors have a high prevalence in surgery and management of home medication is strongly involved in these errors. In scheduled surgery, the preoperative consultation is a privileged time to inform the patient about the management of her/his home medication before admission. This study assessed the impact of a pre-anesthesia best possible medication history (PA-BPMH) on admission. The PA-BPMH was performed by a clinical pharmacist prior to the anesthesia consultation for anesthesiologists to prescribe admission medical orders for scheduled orthopedic surgery patients.
\end{abstract}

Methods: This was a prospective observational study which was carried out in an orthopedic surgery department. All patients over 18 years old with an elective orthopedic surgery were eligible except ambulatory surgery patients. The pharmacist registered the PA-BPMH into the software making it available for anesthesiologists for the preadmission medication order. Finally, a medication reconciliation was performed at admission. The main outcome was the percentage of patients with at least one unintended medication discrepancy (UMD) at admission. The nature, potential clinical impact and acceptance rate of each UMD detected were assessed. Also, the PA-BPMH process was described and patients and anesthesiologists satisfaction was evaluated.

Results: A total of 455 patients had a pharmaceutical consultation. Medication reconciliation was performed at admission for 360 patients. Overall, at least one UMD was observed in $13.0 \%$ of patients ( $n=47)$. A total of 63 UMD were detected. The most common type of UMD was omission (25.4\%) and incorrect drug (23.8\%).Two UMD (3.2\%) were evaluated as life threatening. All the UMD detected were corrected on the admission medication order.

Conclusion: A preoperative pharmacist-anesthesiologist teamwork seems to improve the safety of perioperative management of home medication for scheduled orthopedic surgery patients. This process needs a randomized clinical trial across a wider range of surgeries before its implementation.

Keywords: Pharmacy, Surgery, Anesthesia, Medication errors, Medication reconciliation, Drug safety

\footnotetext{
*Correspondence: amelie.renaudin@chu-nimes.fr

'Department of Pharmacy, Nimes University Hospital, University of Montpellier, Place du Professeur Robert Debré, 30029 Nîmes Cedex 9, France

Full list of author information is available at the end of the article
} 


\section{Background}

Adverse drug events (ADE) are a public health problem. Medication management in healthcare institutions has become a major challenge. In fact, ADE occur in 20 to $72 \%$ of hospitalizations [1-3] and are responsible for 9.7\% of permanent disability [4]. They represent a cost of $\$ 2595$ to $\$ 4685$ per $\mathrm{ADE}$ [5]. In order to reduce these errors, the medication reconciliation (MR) has been developed. Considered by the World Health Organization important for achieving medication safety, MR is the process of identifying and resolving medication discrepancies to prevent ADE at interfaces of care [6]. The MR is used to compare the best possible medication history (BPMH) and the current admission medication order (AMO) to identify and solve unintended medication discrepancies (UMD) [7, 8].

In surgical departments, the incidence of medication errors is higher than in medical departments $(24.3 \%$ vs. 43\%) [9-11]. Indeed, drug management is complex with multiple actors and several places of prescription over often short periods of inpatient stay, increasing the potential risk of medication errors [12]. The roles of each actor, surgeons and anesthesiologists, should be defined for the medication order and the perioperative management of home medication in wards.

In scheduled surgery, the most common medication error found is the omission of home medication [13-16]. In their study, Haley et al. found that $60.3 \%$ of patients had at least one medication error related to usual treatment and $43.3 \%$ of these errors were considered potentially harmful [17]. In Nîmes orthopaedic surgery department, anesthesiologists prescribe medicines to scheduled patients during the anesthesia consultation. Nevertheless, a three-month study showed that $53 \%$ of patients $(n=215)$ had at least one UMD detected at admission by a clinical pharmacist (unpuplished data: preliminary study).

Thus, a pre-anesthesia best possible medication history (PA-BPMH) established by a pharmacist prior to the anesthesia consultation has been implemented in Nimes orthopaedic surgery department. It is available for anesthesiologists for the pre-admission medication order. Indeed, only with an accurate medication history can decisions be made safely regarding the perioperative management of medications.

The primary objective of our study was to assess the impact of PA-BPMH performed by a clinical pharmacist prior to the anesthesia consultation of scheduled orthopedic surgery patients on the number of UMD at admission. Secondary objectives were to characterize UMD and describe the PA-BPMH process.

\section{Methods}

\section{Study population}

This was a prospective observational single centre study which was carried out in a French university hospital in an orthopedic surgery department. The study was performed from June 2018 to February 2019. All patients over 18 years old with an elective orthopedic surgery were eligible. We excluded ambulatory surgery patients and those who already had an anesthesia consultation in another hospital. All methods were carried out in accordance with relevant guidelines and regulations. Informed consent was obtained from all subjects and/or their legal guardians. This study was a practice assessment, so verbal informed consent was obtained from all subjects and/or their legal guardians. According to French law, an Institutional review board (IRB) (number $\left(\mathrm{N}^{\circ} 18.07 .01\right)$ was granted by the Ethics Committee of the Nimes hospital center.

\section{Study design}

Around 1 month before surgery, patients had an appointment for a pharmaceutical consultation $20 \mathrm{~min}$ before the anesthesia consultation. The pharmacist established a pre-anesthesia best possible medication history (PA-BPMH) available for the anesthesiologist. During their consultation, anesthesiologists prescribed medication orders using PA-BPMH.

The study design was carried out in 3 phases described below in Fig. 1.

\section{Phase 1: pharmaceutical consultation preparation}

One week before consultation, the pharmacist prepared the PA-BPMH using the electronic hospital scheduling system. First, the pharmacist checked all medical information in the patient medical records and called the community pharmacy who transmitted medication orders. The pharmacist then transcribed them into the hospital prescription support software.

\section{Phase 2: pharmaceutical consultation}

During consultation, the pharmacist checked all medication data collected with the patient and made proposals for therapeutic equivalences if needed. The pharmacist used the 'Vitale' microchip patient card, when available. This card contains a pharmaceutical record fulling from the community pharmacist with dispensed medication history during the last 4 months. The pharmacist registered the PA-BPMH into the software making it available for anesthesiologists for the pre-admission medication order.

The patient satisfaction was evaluated by anonymous self-assessment survey after each pharmaceutical consultation. At the end of the study, the satisfaction of each anesthesiologist using the PA-BPMH was also collected. 




Fig. 1 Study design. PA-BPMH: pre-anesthesia best possible medication history, MR: medication reconciliation

\section{Phase 3: medication reconciliation at admission}

At patient admission, to avoid contamination bias, a medication reconciliation was performed within $24 \mathrm{~h}$ by a different clinical pharmacist than the one at the preadmission phase. The pharmacist established a BPMH and compared it with patient's admission medication order. He detected and resolved UMD with the physician.

\section{Outcome measures}

The primary outcome was to assess the percentage of patients conciliated at admission with at least one UMD.

Among the secondary outcomes, the nature, potential clinical impact and acceptance rate of each UMD detected were assessed. For clinical impact, methodology used was blind retrospectively evaluation by a senior clinical pharmacist (different from investigators) and a senior anesthesiologist by using the method described by Quélennec et al. [18], a European adaptation of the National Coordinating Council for Medication Error Reporting and Prevention (NCC MERP) Index [19].

Other secondary outcomes were: (1) the time spent at preparation (phase 1) and pharmaceutical consultation (phase 2); (2) type and number of sources for performing PA-BPMH; (3) the rate of therapeutic equivalences proposed by the clinical pharmacist; (4) the satisfaction assessment of patients and anesthesiologists.

For each patient with a complete process (pharmaceutical consultation and medication reconciliation), basic demographical data was collected (age, gender, type of surgery, length of hospital stay) as well as the time between pharmaceutical consultation and patient admission.

\section{Statistical analysis}

Descriptive statistics were reported as counts and percentages for categorical variables, means and standard deviations for continuous variables with normal distribution, and median and quartiles for others. All data collected were entered into REDCap ${ }^{\circ}$ software (V8.5.1,
2018) [20] and all statistical analyses were performed using Microsoft Excel ${ }^{\circledR}$ software (V2016).

\section{Results}

\section{Patient characteristics}

During the study period, 455 patients had a pharmaceutical consultation. Thereafter, medication reconciliation was performed at admission for 360 patients (Fig. 2). Among these patients, the median age was 68.0 years old $(\mathrm{IQR}=[59.0 ; 75.0]$.$) and 54.0 \%$ were female (Table 1$)$. The majority of the study population $(83.4 \%)$ had home medication and the average number of pre-admission medications was $4.4 \pm 3.7$ per patient. The median time between the anesthesia consultation and surgery admission was 27 days $(\mathrm{IQR}=[20.0 ; 30.0]$.

\section{Characterisation of UMD}

Medication reconciliation was performed within $24 \mathrm{~h}$ after admission for $87.8 \%$ of patients $(n=360)$ who had

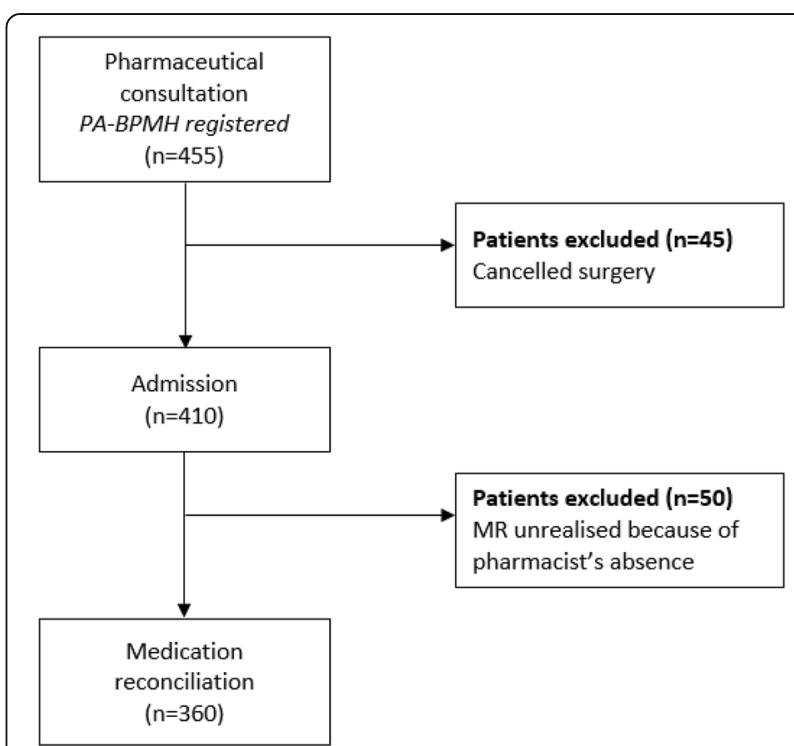

Fig. 2 Flow chart. PA-BPMH: pre-anesthesia best possible medication history, MR: medication reconciliation 
Table 1 Baseline characteristics of the study population

\begin{tabular}{ll}
\hline Characteristics & Patients $(n=360)$ \\
\hline Age $(y r)$ & $68[59.0 ; 75.0]$ \\
Sex & $193(54 \%)$ \\
Female & $167(46 \%)$ \\
$\quad$ Male & \\
Surgical indication & $141(39.2 \%)$ \\
$\quad$ Knee arthroplasty & $108(30.0 \%)$ \\
Hip arthroplasty & $33(9.1 \%)$ \\
Spine surgery & $78(21.7 \%)$ \\
$\quad$ Other surgery & $4[1.0 ; 7.0]$ \\
Number of lines of PA-BPMH & $4[4.0 ; 6.0]$ \\
Length of stay (days) &
\end{tabular}

a pharmaceutical consultation. At least one UMD was observed in $13.0 \%$ of patients $(n=47)$. All the UMD detected were corrected on the AMO.

The pharmacist detected 63 UMD, with a mean of $1.3 \pm 0.7$ UMD per patient. Among these 47 patients, $74.5 \%$ had one UMD, $19.1 \%$ had 2 UMD and $6.4 \%$ had 3 to 5 UMD. The most common type of UMD was omission $(25.4 \%)$ and incorrect drug (23.8\%) or dose $(23.8 \%)$ (Table 2). The most frequent medications involved belonged to the cardiovascular system (34.9\%) (Table 2), including anticoagulants (9.1\%), angiotensin-converting enzyme inhibitors (13.6\%) and angiotensin receptor blockers (18.2\%).

Discrepancies were classified into three categories: level 1 no potential harm (NCC MERP category C), level 2 monitoring or intervention potentially required to preclude harm (NCC MERP category D) and level 3 potential harm (NCC MERP categories D and above) $(16,17)$.

Among the $63 \mathrm{UMD}, 38.1 \%(n=24)$ errors were classified as level $1,30.2 \%(n=19)$ as level 2 and $31.7 \%(n=$ 20) as level 3. Two UMD (3.2\%) were life threatening involving an anticoagulant and an antiarrhythmic.

\section{Pharmaceutical consultation process}

The median time to prepare PA-BPMH (phase 1) was $17.0 \mathrm{~min}(\mathrm{IQR}=[12.0 ; 21.0])$ and for the consultation itself (phase 2) was $6.0 \mathrm{~min}(\mathrm{IQR}=[4.0 ; 11.0])$. The main information sources for performing $\mathrm{PA}-\mathrm{BPMH}$ during phase 1 and phase 2 were: patient (100\%), medical record $(100 \%)$, community pharmacist $(94.8 \%)$ and pharmaceutical record (57.1\%). The median number of information sources was $4.0(\mathrm{IQR}=[4.0 ; 5.0])$. Also, to optimize medication management, when the home medication was unavailable at the hospital, the pharmacist proposed $268(12.1 \%)$ therapeutic equivalences on 2221 lines of PA-BPMH.

\section{Patient and anesthesiologist satisfaction}

Overall, $45.7 \%$ of patient surveys were filled in $(n=455)$. All patients found the pharmaceutical consultation useful and were able to better understand their medication management at the hospital. Also, the pharmacist's welcome was considered very satisfactory by $92.3 \%$ of them.

All anesthesiologists (12 physicians) completed the survey and all found that the pharmaceutical consultation was helpful for their activity, improved the management of home medication safety, ensured better perioperative medication management, and saved time. They all believed the pharmaceutical consultation must be continued.

\section{Discussion}

This study assessed the impact of PA-BPMH on the number of UMD at admission. The PA-BPMH was performed by a clinical pharmacist prior to the anesthesia consultation for anesthesiologists to prescribe admission medical orders for scheduled orthopedic surgery patients.

At least one UMD was observed in $13.0 \%$ of patients and all corrected by physicians at admission. Previous studies have shown that medication discrepancies at admission can be decreased with the help of a clinical pharmacist before patient admission [14, 21, 22]. Indeed, without this help, studies estimate that 48.8 to $60.3 \%$ of patients have at least one UMD at surgery admission $[17,23,24]$. These results are consistent with our previous study that found $53 \%$ of patient had at least one UMD without PA-BPMH.

Table 2 Unintentional medication discrepancies grouped according to ATC and type of discrepancy

\begin{tabular}{lllllll}
\hline ATC & Omission & Incorrect dose & Incorrect drug & No indication & Incorrect frequency & Total UMD \\
\hline C & $4(25.0 \%)$ & $4(26.6 \%)$ & $5(33.3 \%)$ & $4(36.4 \%)$ & $5(83.3 \%)$ & $22(34.9 \%)$ \\
N & $2(12.5 \%)$ & $7(46.7 \%)$ & $4(26.7 \%)$ & $3(27.2 \%)$ & $0(0.0 \%)$ & $16(25.4 \%)$ \\
R & $2(12.5 \%)$ & $0(0.0 \%)$ & $3(20 \%)$ & $2(18.2 \%)$ & $0(0.0 \%)$ & $7(11.1 \%)$ \\
$J$ & $4(25.0 \%)$ & $1(6.7 \%)$ & $1(6.7 \%)$ & $0(0.0 \%)$ & $0(0.0 \%)$ & $6(9.5 \%)$ \\
A & $2(12.5 \%)$ & $1(6.7 \%)$ & $0(0.0 \%)$ & $2(18.2 \%)$ & $0.0 \%)$ & $5(8.0 \%)$ \\
Others & $2(12.5 \%)$ & $2(13.3 \%)$ & $2(13.3 \%)$ & $0(0.0 \%)$ & $1(16.7 \%)$ & $7(11.1 \%)$ \\
Total UMD & $16(25.4 \%)$ & $15(23.8 \%)$ & $15(23.8 \%)$ & $11(17.5 \%)$ & $6(9.5 \%)$ & 63 \\
\hline
\end{tabular}


Among the strengths of our study, to our knowledge, our process is the first model of a clinical pharmacistanesthesiologist teamwork consulting in the same unit of place and time. This collaboration optimizes the management of home medication safety for scheduled orthopedic surgery patients. Indeed, the PA-BPMH typically takes the pharmacist $23 \mathrm{~min}$ and they cross-reference the patient reported information with four information sources to ensure an accurate and complete medication history. The pharmacists seems to be the ideal actors to establish the PA-BPMH because of their focus on medication and their knowledge about it $[14,15]$. Also, this pilot study had sufficient patients included $(n=360)$, as well as the observational period ( 8 months) to ensure its routine implementation is feasible.

Among the UMD, the most common was medication omission as identified in previous studies [22-25]. These UMD were related with medication additions or modifications by other physicians (mostly antihypertensive medication) occurring between anesthesiologist consultation and admission, for example, when patients have the cardiology consultation during this period. These medication modifications are unavoidable and require that any change to the home medication must be checked at admission.

In addition, by placing a pharmacist upstream in the care process, others benefits are expected. Our team of anesthesiologists found that the pharmaceutical consultation was useful, permitting a better perioperative medication management and saving time during their consultation. Also, the pharmacist was very welcomed by most of patients who found the pharmaceutical consultation useful to better understand their home medication management at admission.

The software is a key point of our process. It has permitted that the pharmacist to enter the PA-BPMH making it available for anesthesiologists for the preadmission medication order. Adequate information technology avoids the errors of transcription order and preserves the veracity of the original information. In fact, for Orser et al [26], with information technology, it is possible to share information and it facilitates coordination between health professionals.

Our study has some limitations. To strengthen the study methodology, a prospective analysis using randomized selection into control and intervention groups should be performed. Tested in scheduled orthopedic surgery, the impact of a pharmaceutical consultation should be extended to other surgical specialties to corroborate our results. In addition, the effectiveness of this activity should be assessed to provide arguments for decision-makers to implement this pharmaceutical consultation. It is also relevant to assess the impact of this model of a clinical pharmacist-anesthesiologist teamwork on clinical patients' outcomes such as mortality, readmission and $\mathrm{ADE}$ occurrence.

\section{Conclusion}

The preoperative pharmaceutical consultation in scheduled orthopedic surgery combined with the anesthesiologist in a teamwork seems to have a positive impact on reduction of unintended medication discrepancies. Further testing of this process across a wider range of surgeries is needed before it can be widely implemented and so we have designed a randomized clinical trial for that purpose (Clinical Trial PREVEMCA NCT04013061). Implementing a pharmaceutical consultation prior to the anesthesia consultation for all scheduled surgeries should be a safe way to reduce significantly the rate of medication errors.

\section{Abbreviations}

ADE: Adverse drug events; AMO: Admission medication order; ATC: Anatomical therapeutic chemical classification system; BPMH: Best possible medication history; IRB: Institutional review board; MR: Medication reconciliation; NCC MERP: National Coordinating Council for Medication Error Reporting and Prevention; PA-BPMH: Pre-anesthesia best possible medication history; UMD: Unintended medication discrepancy

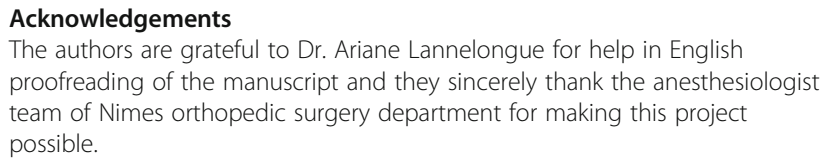
proofreading of the manuscript and they sincerely thank the anesthesiologist team of Nimes orthopedic surgery department for making this project possible.

\section{Authors' contributions}

AR recruited the patients, drafted the case report form, performed the preanesthesia best possible medication histories and the pharmaceutical consultations, collected and controlled the data, wrote the article. GLB co-wrote and corrected the protocol, wrote the methodology of the study, performed statistical analysis, co-wrote and corrected the article. CC assessed the potential clinical impact of each error detected, co-wrote and corrected the article. $\mathrm{AL}$ assessed the potential clinical impact of each error detected, performed the anesthesia consultation, co-wrote and corrected the article. CB co-wrote and corrected the protocol, performed the anesthesia consultations, cOwrote and corrected the article. JMK co-wrote and corrected the protocol, co-planned the study, co-wrote and corrected the article. PC co-wrote and corrected the protocol, co-planned the study, performed the anesthesia consultations, co-wrote and corrected the article. HR wrote the protocol, planned the study, co-wrote and corrected the case report form, performed the medication reconciliations at admission in surgery ward, co-wrote and corrected the article. All authors have read and approved the manuscript.

\section{Funding}

This research did not receive any specific grant from funding agencies in the public, commercial, or not-for-profit sectors.

\section{Availability of data and materials}

The datasets used and/or analysed during the current study are available from the corresponding author on reasonable request.

\section{Ethics approval and consent to participate}

This study was a practice assessment that was reviewed by the Ethics Committee of the Nimes hospital center and received its ethical approval. According to French law, an Institutional review board (IRB) (number $\left(\mathrm{N}^{\circ} 18.07 .01\right)$ was granted. The Ethics Committee of the Nimes hospital center also approved the procedure for obtaining informed verbal consent to participate in this study. Informed verbal consent to participate was obtained from each participant prior to conducting this study. 


\section{Consent for publication}

Not applicable.

\section{Competing interests}

The authors declare that they have no competing interests.

\section{Author details}

'Department of Pharmacy, Nimes University Hospital, University of Montpellier, Place du Professeur Robert Debré, 30029 Nîmes Cedex 9, France. ${ }^{2}$ UPRES EA2415, Laboratory of Biostatistics, Epidemiology, Clinical Research and Health Economics, Clinical Research University Institute, University of Montpellier, Montpellier, France. ${ }^{3}$ Department of Law and Health Economics, Faculty of Pharmacy, University of Montpellier, Montpellier, France. ${ }^{4}$ Department of Anesthesia, Nimes University Hospital, University of Montpellier, Nimes, France. ${ }^{5}$ Department of Anesthesia, Montpellier Cancer Institute (ICM), University of Montpellier, Montpellier, France.

Received: 7 April 2020 Accepted: 4 August 2020

Published online: 13 August 2020

\section{References}

1. Forster AJ, Murff HJ, Peterson JF, Gandhi TK, Bates DW. The incidence and severity of adverse events affecting patients after discharge from the hospital. Ann Intern Med. 2003;138(3):161-7. https://doi.org/10.7326/00034819-138-3-200302040-00007.

2. Forster AJ, Clark HD, Menard A, et al. Adverse events among medical patients after discharge from hospital. CMAJ Can Med Assoc J J Assoc Medicale Can. 2004;170(3):345-9.

3. Baker GR, Norton PG, Flintoft V, et al. The Canadian adverse events study: the incidence of adverse events among hospital patients in Canada. CMAJ Can Med Assoc J J Assoc Medicale Can. 2004;170(11):1678-86.

4. Thomas EJ, Studdert DM, Burstin HR, et al. Incidence and types of adverse events and negligent care in Utah and Colorado. Med Care. 2000;38(3):26171. https://doi.org/10.1097/00005650-200003000-00003.

5. Bates DW, Spell N, Cullen DJ, et al. The costs of adverse drug events in hospitalized patients. Adverse drug events prevention study group. JAMA. 1997;277(4):307-11.

6. Haute Autorité de Santé. Mettre en œuvre la conciliation des traitements médicamenteux en établissement de santé. Available from URL: https:// www.has-sante.fr/portail/jcms/c_2736453/fr/mettre-en-oeuvre-laconciliation-des-traitements-medicamenteux-en-etablissement-de-sante [Accessed Dec 2018].

7. Institute for Safe Medication Practices Canada. Medication Reconciliation. https://www.ismp-canada.org/medrec/ [Accessed Dec 2018].

8. Gleason KM, Brake H, Agramonte V, Perfetti C. Medications at transitions and clinical handoffs (MATCH) toolkit for medication reconciliation. (prepared by the island peer review organization, Inc, under contract no. HHSA2902009000 13C.) AHRQ publication no. 11(12)0059. Agency for Healthcare Research and Quality: Rockville; 2012.

9. Fijn R, Van den Bemt PMLA, Chow M, De Blaey CJ, De Jong-Van den Berg LTW, Brouwers JRBJ. Hospital prescribing errors: epidemiological assessment of predictors. Br J Clin Pharmacol. 2002;53(3):326-31. https://doi.org/10. 1046/2Fi.0306-5251.2001.bjcp1558.doc.x.

10. Unroe KT, Pfeiffenberger T, Riegelhaupt S, Jastrzembski J, Lokhnygina $Y$, Colón-Emeric C. Inpatient medication reconciliation at admission and discharge: a retrospective cohort study of age and other risk factors for medication discrepancies. Am J Geriatr Pharmacother. 2010;8(2):115-26. https://doi.org/10.1016/j.amjopharm.2010.04.002.

11. Rentero L, Iniesta C, Urbieta E, Madrigal M, Pérez MD. Causas y factores asociados a los errores de conciliación en servicios médicos y quirúrgicos. Farm Hosp. 2014;38(5):398-404. https://doi.org/10.7399/fh.2014.38.5.1136.

12. Haute Autorité de Santé. Circuit du médicament en chirurgie programmée. Available from URL: https://www.has-sante.fr/portail/jcms/c_834650/fr/ circuit-du-medicament-en-chirurgie-programmee [Accessed Dec 2018].

13. Tran T, Taylor SE, Hardidge A, et al. The prevalence and nature of medication errors and adverse events related to preadmission medications when patients are admitted to an orthopedic inpatient unit: an observational study. Ann Pharmacother. 2019:53(3):252-60. https://doi.org/ $10.1177 / 1060028018802472$.
14. Kwan Y, Fernandez OA, Nagge JJ, et al. Pharmacist medication assessments in a surgical preadmission. Clinic Arch Intern Med. 2007;167(10):1034. https://doi.org/10.1001/archinte.167.10.1034.

15. Quennery S, Cornu O, Sneyers B, Yombi JC. Added value of pharmacistacquired drug histories in an orthopaedic ward. Acta Clin Belg. 2011;66(3): 196-9. https://doi.org/10.2143/ACB.66.3.2062546.

16. Duthie DJR, Montgomery JN, Spence AA, Nimmo WS. Concurrent drug therapy in patients undergoing surgery. Anaesthesia. 1987:42(3):305-6. https://doi.org/10.1111/j.1365-2044.1987.tb03045.x.

17. Haley M, Raymond C, Nishi C, Bohm E. Drug-related problems in patients undergoing elective Total joint Arthroplasty of the hip or knee. Can J Hosp Pharm. 2009;62(5):360-6. https://doi.org/10.4212/cjhp.v62i5.822.

18. Quélennec B, Beretz L, Paya D, et al. Potential clinical impact of medication discrepancies at hospital admission. Eur J Intern Med. 2013;24(6):530-5. https://doi.org/10.1016/j.ejim.2013.02.007.

19. National Coordination Council for Medication Error Reporting and Prevention. Types of Medication Errors 2001. Available from URL: https:// www.nccmerp.org/types-medication-errors (Accessed Mars 2019).

20. Harris PA, Taylor R, Thielke R, Payne J, Gonzalez N, Conde JG. Research electronic data capture (REDCap) - a metadatadriven methodology and workflow process for providing translational research informatics support. J Biomed Inform. 2009:42(2):377-81.

21. Van den Bemt PM, van den Broek S, van Nunen AK, Harbers JB, Lenderink AW. Medication reconciliation performed by pharmacy technicians at the time of preoperative screening. Ann Pharmacother. 2009;43(5):868-74. https://doi.org/10.1345/aph.1L579.

22. Leung V, Mach K, Charlesworth E, Hicks S, Kizemchuk K, Stumpo C. Perioperative medication management (POMM) pilot: integrating a community-based medication history (MedsCheck) into medication reconciliation for elective orthopedic surgery inpatients. Can Pharm J Rev Pharm Can. 2010;143(2):82-7. https://doi.org/10.3821/2F1913-701X-143.2.82.

23. Fummi C, Brunschweiler B, Vacher $\mathrm{H}$. Conciliation médicamenteuse à l'admission du patient dans une unité de chirurgie orthopédique septique: une étude menée sur six mois 2015;34:7. https://doi.org/10.1684/jpc.2015.0319.

24. Pascual O, Real JM, Uriarte M, Larrodé I, Alonso YM, Abad MR. Evaluation of medication reconciliation in a trauma unit. Rev Esp Cir Ortopédica Traumatol Engl Ed. 2015:59(2):91-6. https://doi.org/10.1016/.jecot.2014.07.003.

25. González-García L, Salmerón-García A, García-Lirola M, Moya-Roldán S, Belda-Rustarazo S, Cabeza-Barrera J. Medication reconciliation at admission to surgical departments: medication reconciliation at admission. J Eval Clin Pract. 2016;22(1):20-5. https://doi.org/10.1111/jep.12403.

26. Orser BA, Hyland S. U D, Sheppard I, Wilson CR. Review article: improving drug safety for patients undergoing anesthesia and surgery. Can J Anesth Can Anesth. 2013;60(2):127-35. https://doi.org/10.1007/s12630-012-9853-y.

\section{Publisher's Note}

Springer Nature remains neutral with regard to jurisdictional claims in published maps and institutional affiliations.

\section{Ready to submit your research? Choose BMC and benefit from:}

- fast, convenient online submission

- thorough peer review by experienced researchers in your field

- rapid publication on acceptance

- support for research data, including large and complex data types

- gold Open Access which fosters wider collaboration and increased citations

- maximum visibility for your research: over $100 \mathrm{M}$ website views per year

At BMC, research is always in progress.

Learn more biomedcentral.com/submission 